\title{
An Open Design for an Air Flow and Pressure Control Circuit for Medical Devices
}

\author{
Mike Teodorescu ${ }^{\mathrm{a}, \mathrm{b}}$ \\ ${ }^{\text {a } I n f o r m a t i o n ~ S y s t e m s ~ D e p a r t m e n t, ~ B o s t o n ~ C o l l e g e ~}$ \\ ${ }^{b}$ Research Affiliate, D-Lab, Massachusetts Institute \\ of Technology \\ Cambridge Massachusetts, U.S.A. \\ mike.teodorescu@bc.edu,hmteodor@mit.edu
}

\author{
Horia-Nicolai Teodorescu a,b \\ a Lab. BME, 'Gh. Asachi' Technical University of \\ Iasi \\ ${ }^{\mathrm{b}}$ Institute of Computer Science, RO Academy \\ Iasi, Romania \\ hteodor@etti.tuiasi.ro
}

\begin{abstract}
We report on an air control system with low power consumption for small enclosures with mainly medical applications, such as lung ventilators, APAP machines, PAPR isolation hoods, surgical applications, and aerosol containment. The circuit shared can be built from cost effective, commercially available parts; it represents a pressure control system for positive pressure environments such as hoods used in personal protection equipment (PPE), as proposed in today's COVID-19 epidemic. An essential feature of the approach is that the circuit uses advanced off the shelf modules that include microcontrollers, but the overall design remains analogic. Another contribution is to derive rigorous design equations that bring together the pneumatic and electric nonlinear equations describing an inflatable enclosure, the linearized pneumatic circuits, the power device (fan), and the control loop. We warn that the designs in this paper are for scientific testing only and not for any medical use; no guarantee is implied. See the Warnings and Disclaimer.
\end{abstract}

Keywords-control; air pressure control; open design; low power; nonlinear characteristic; portable equipment; PPE; PAPR; COVID-19.

\section{INTRODUCTION}

Numerous medical and industrial applications require maintaining small closed spaces ventilated and at a small positive pressure. Such devices comprise neo-natal and respiration support ventilators [1], [2] with numerous solutions known in the literature [3]. Continuous positive pressure systems such as CPAP (continuous positive airway pressure) or APAP (automatic positive airway pressure) machines are used in supporting patient airways and are well known in the medical device industry. PAPR isolation hoods are used in environments which require a high level of air filtration for protection of medical staff in situations of infectious aerosolized particles, as is the case of the current COVID-19 pandemic. Sterile enclosures for surgical procedures [4] also require filtered air and positive pressure. All are potential applications for the system described in this paper. Such equipment is in high demand especially during infectious disease events such as the COVID-19 crisis. For instance, a PAPR machine creates positive pressure inside a hood, is battery operated (thus low energy circuitry increases longevity on one charge) and provides HEPA filtered air (High Efficiency Particulate Air) to the wearer. Depending on implementation, the enclosure surrounding the wearer's face can be either a rigid structure covering the entire face of the wearer, a bubble covering the entire head of the wearer (where the positive pressure creates tension in the walls), or a semi-flexible structure with rigid elements embedded to fit around the face of the wearer. The HEPA air filtration system used in a PAPR provides a higher level of efficiency than an N95 respirator. However, PAPR machines are not as commonplace as N95 respirators, as they are more expensive and require servicing between uses (charging and cleaning). The circuit presented in this paper can be built relatively inexpensively from offthe-shelf components and has the advantage of being low-power and providing versatility for multiple device applications: PAPRs, BiPAPs (with minor modifications such as an additional sensor), CPAPs, and inflatable positive pressure enclosures which require filtered air and a constant pressure maintained.

There are various requirements for pressure and flow controllers in medical devices, depending on the device using the controller. Ventilators for human use are typically intensive care unit (ICU) devices that are connected to the main power supply [5] which thus do not benefit as much from a low-power system as described here. In the design reported here, the main constraint is power consumption as we are looking at battery-operated systems such as PAPRs. An issue is the use of efficient air filters for restraining aerosols such as viruses [6], as such filters have a considerable pressure drop on them. Additional consideration in the design is given to portability, as patient isolation equipment as well as personal protection equipment (PPE) is battery powered.

We discuss the design of a system that maintains a small space for field-controlled atmosphere operations such as collection of samples without contamination, safe collection of biological samples, respiratory support, or surgery. The system has several requirements, including minimal power requirement of battery-operated systems, and medical standards. In addition to the above, the weight and dimensions of the control system are critical.

Section II presents the main ideas of the circuit design using advanced off the shelf modules that include microcontrollers, yet the overall design remaining analogic. Section III introduces the detailed nonlinear pneumatic and electric equations describing the system and shows how the design can be optimized. The last section is conclusive. 


\section{The BASIC CONTROL CIRCUIT}

There are essentially two types of controllers in pneumatics, namely gas flow controllers and gas pressure controllers. The difference between them is that the first type can maintain a constant flow when the pressure changes, while the second type varies the gas flow with the aim of maintaining a constant pressure in an enclosed space, such as a hood part of a respirator system, or a CPAP airway, or an isolation enclosure (used for example for patient transport, surgery, or intubation). The presented system belongs to the pressure control devices, yet it allows for a compromise between a minimal flow rate and a minimal pressure maintained in the enclosure. In a CPAP application for example, a minimum pressure is needed to maintain airway support of the patient. In a PAPR example, a minimal flow is necessary to ensure filtration of potentially contaminated outside air. In an inflatable application, such as a patient isolator or a surgical enclosure [4], where self-support of the enclosure is needed, there are apparently contradictory requirements: there is a minimal pressure that maintains the integrity of the self-inflated enclosure structure and a minimal positive pressure that ensures that contaminated environmental air does not enter the enclosure when a port of the enclosure is opened; at the same time, regulations for surgery require a minimum number of air changes per hour, leading to a minimal flow requirement.

To simplify the circuitry and thus reduce circuitry weight, dimensions and power consumption, while increasing the circuit reliability, a circuit was designed that has a very simple structure yet making use of recent technologies available. The electronic system implements three major functions: (a) power supply protection for the entire system (reverse voltage, overcurrent, overvoltage), (b) DC-DC, 12 to $5 \mathrm{~V}$ conversion, power supply regulation, and protection for the $5 \mathrm{~V}$ circuit section, (c) differential pressure measurement, voltage-controlled PWM generation, and power control of the fan. The power supply protection for the entire system is performed by the integrated circuit MAX17561. It ensures a lower voltage drop than a Schottky diode, even at high currents (7 A). With a Low $\mathrm{R}_{\mathrm{ON}}$ of Typically) $100 \mathrm{~m} \Omega$, the circuit is saving about $0.2 \mathrm{~V}$ allowing the motor to operate at higher voltages. This extends the capability of the fan at large air flows. The DC-DC (12 to $5 \mathrm{~V}$ ) conversion and power supply regulation and protection is done with MAX17502 circuit.

A critical element is the intelligent sensor for differential pressure measurement with temperature compensation and conversion of the pressure in proportional voltage (ratiometric conversion). In the first configuration of control system discussed, the second critical circuit is the voltage-controlled PWM generator circuit LTC6992-X. This circuit receives the voltage signal from the sensor and produces a PWM modulation with 0 to $100 \%$ pulse width. The signal from this circuit drives the output power MOSFET that controls the average voltage on the motor of the fan, $\left\langle u_{f a n}\right\rangle=\eta V_{c c}$, where $\eta$ is the pulse width over period ratio. The overall diagram is shown in Fig. 1. The electronic system can also be controlled manually (P1) using a potentiometer. The operation with manual control is suboptimal and the integrator mode is the best use case.

The presented automatic control is not optimal, in that the pulse width, $\tau=\tau(\Delta P)$, is a linear function in the pressure difference $\Delta \mathrm{P}$, which does not ensure the fastest attainment of $\Delta P=0$, because the air flux (flow) generated is not proportional with the voltage on the fan motor.

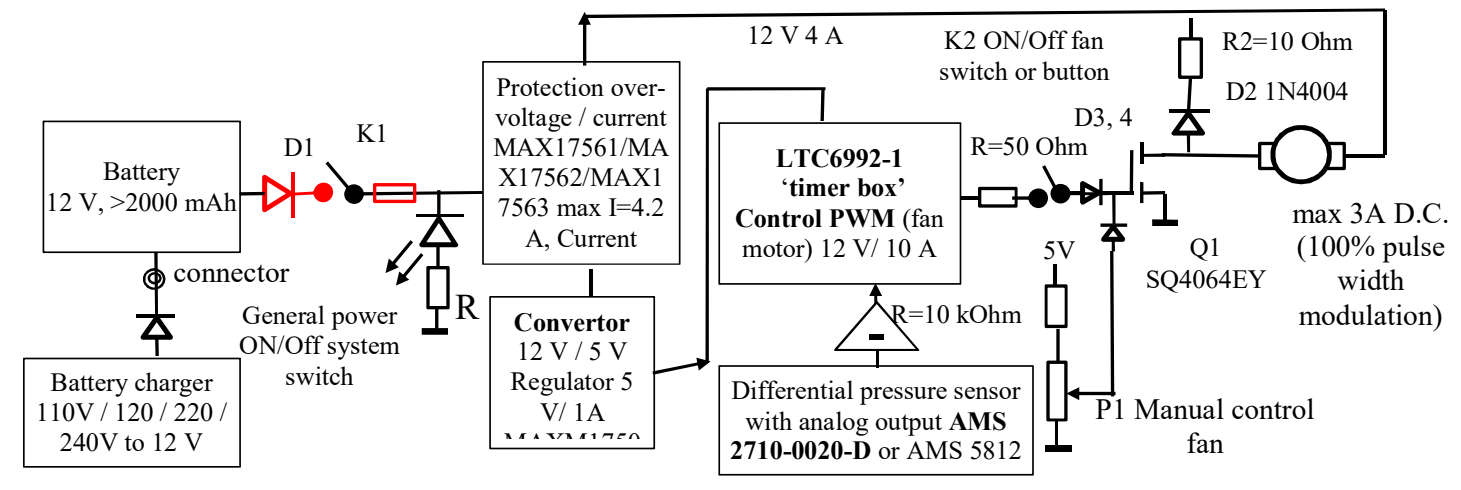

Figure 1. Diagram of a basic versions of the electronic control system

The fuse shown in Fig. 1 is not needed with circuit MAX17561. Also, with the same circuit the reverse voltage protection diode (Schottky barrier rectifier 60 $\mathrm{V}$ reverse minimum, $5 \mathrm{~A}$ ) is not needed. The alternative protection circuits in Fig.1 are commercially available as well.

Various manufacturers provide pressure sensors suitable for the application; such sensors are AMS 5812 - pressure sensor with analog and I2C output, which requires a $\mathrm{VS}=5.0 \mathrm{~V}$ only and produces analog output of up to $4 \mathrm{~V}$, the NPA Series of surfacemount pressure sensors produced by Amphenol.
Differential pressure sensor types tested in second author's laboratory include TruStability ${ }^{\mathrm{TM}}$ board mount pressure sensors RSC Series (Honeywell), MPX5500DP sensor (Motorola), and HSCDRRN2.5MGAB5 (Honeywell; letters mean 2.5 mbar, differential, analog output, $5 \%$ to $95 \%$ of supply voltage output, $5 \mathrm{~V}$ supply). The connection of the ratiometric pressure sensor to the controller is shown in Fig. 2.

The differential pressure measured is between 0 and about 1 bar, with the higher pressure inside the enclosure. This pressure range is transformed by the 
AMS 5812 circuit in a 0 to $4 \mathrm{~V}$ range which is fed to the PWM controller. The last one produces 0 -width pulse for $0 \mathrm{~V}$ and $100 \%$ width for $4 \mathrm{~V}$.

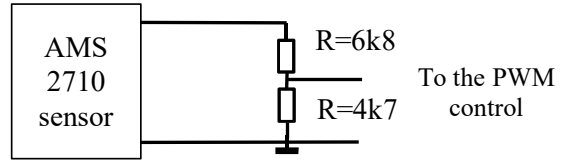

Figure 2. Connecting the AMS ratiometric pressure sensor to the PWM controller.

In the system version shown in Fig. 1, the PWM control system is based on the integrated circuit LTC6992 which is a voltage-controlled pulse width modulator with analog input suitable for the output from the pressure sensor circuit AMS 2710.

The connection between the sensor and the controller includes an inverting amplifier which has also the role of integrator (Fig. 3). The integration function is needed to prevent small accidental pressure variations from minor movement of the enclosure being detected as a true drop of pressure requiring a change in the fan regime. $C_{2}$ removes noise from the reference voltage. The output voltage is $V_{\text {out }}=\frac{R 1}{R 2}$. $\left(V_{\text {ref }}-V_{\text {in }}\right)$, where $V_{\text {ref }}$ depends on the maximal pressure because $V_{i n-m a}$ is given by $5 \mathrm{~V} \times \frac{\mathrm{P}_{\text {max real }}}{\mathrm{P}_{\text {maxsensor }}}$. As the minimal value of $V_{\text {out }}$ corresponds to the minimal required flow, $V_{\text {out }- \text { min }}=\frac{R 1}{R 2} \cdot\left(V_{\text {ref }}-5 \mathrm{~V} \times\right.$ $\left.\frac{P_{\max \text { real }}}{P_{\text {max sensor }}}\right)$.

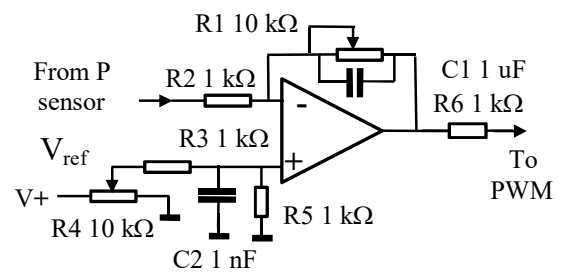

Figure 3. Inverting amplifier also used as integrator and as PWM range modifier

$V_{\text {ref }}$ is determined from the condition $V_{\text {in }}=0$ when $V_{\text {out }}$ would be for example $4.5 \mathrm{~V}$, so $V_{\text {ref }}=$ $4.5 \mathrm{~V} \cdot \frac{R 1}{R 2}$. The adjustable reference allows choosing a realistic value for the maximal pressure in the enclosure. The adjustable gain $\frac{R 1}{R 2}$ provides flexibility in the control of the PWM duty cycle.

Because the system with the active control represents a closed loop with positive feedback, with the behavior (frequency characteristic) of the enclosure essentially unknown, there is a chance that the loop oscillates, with perceptible result consisting in vibrations of the air duct and with fluctuations in the pressure in the enclosure. To avoid vibrations when the air flow changes rapidly, the capacitor $\mathrm{C} 1$ in Fig. 3 may need a larger value to dampen such oscillations; the value must be adapted to the specific application used.

The system is damped mainly because of the air friction in the ducts and in the enclosure, but the damping is unknown. While it would be easy to increase the damping of the ducts by using air traps and turns, it is undesirable, because the power consumption would increase. The requirements of power consumption and of lack of vibrations are conflicting. The solution is to use an integrative control, with the amplifier turned into an integrating amplifier using a capacitor in the amplifier feedback loop. The time constant of the group $R_{1} C_{1}$ is $1 C 1=$ $10^{4} \cdot 10^{-6}=10 \mathrm{~ms}$. This means that oscillations of the pressure with frequencies above $100 \mathrm{~Hz}$ will be damped. However, the manufactured system must be tested under various conditions, with a person manipulating the enclosure with the hands in the gloves and checking if oscillations/vibrations occur. If so, the value of $C_{1}$ should be increased, for example $10 \mu F$ or even larger.

The fan 'San Ace 60 model 9CR0612S001' is a good choice for such applications, with rated voltage $12 \mathrm{~V}$, operating range 10.8 to $13.2 \mathrm{~V}$, rated (nominal) current 3.2 A, maximal air flow $2.26 \mathrm{~m}^{3} / \mathrm{min}$, max air pressure $550 \mathrm{~Pa}$, and reasonable noise $66 \mathrm{dBA}$. The PWM controller San ACE (Sanyo Denki America Inc.), type 9PC8666X-S001, $12 \mathrm{~V}$ is a good choice for replacing the LTC6992-1 'timer box' and the power MOSFET, allowing fast prototyping when using San Ace fans. The module produces an output current of $20 \mathrm{~mA}$ max, and is able to drive up to 4 fans. This allows another design, which is more compact. The second version of the control system is sketched in Fig. 4, without the sensor circuitry.
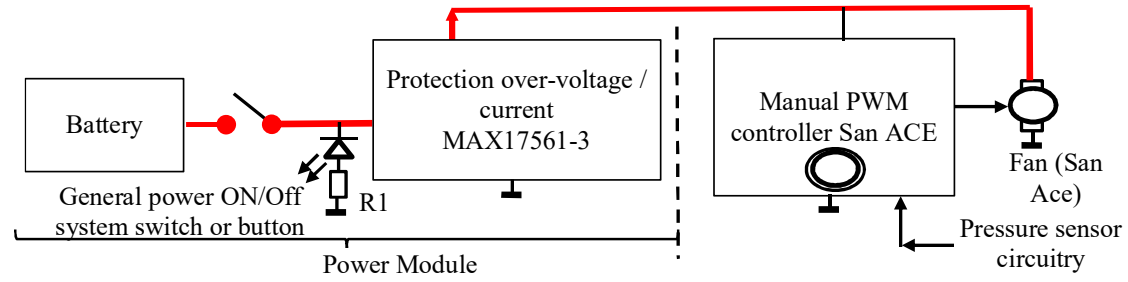

Figure 4. Second version of the control system, without the sensor circuitry.

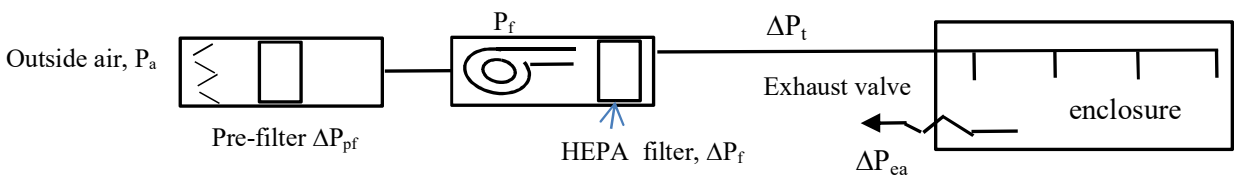

Figure 5. Basic pneumatic scheme of a HEPA filtered application. When a pre-filter is used, a pressure difference on it appears, $\Delta \mathrm{P}_{\mathrm{pf}} . \Delta \mathrm{Pio}$ is the exhaust valve pressure difference and corresponds to enclosure to ambient pressure difference. 


\section{EQUATIONS GOVERNING THE PNEUMATIC OPERATION}

The operation of the control system has to take into account two intermingled processes, the pneumatic one and the electrical one. The interfaces between these processes are the pressure sensor and the fan with its motor. A combination of pneumatic and electrical equations describes the system. The aim of the theoretical analysis is to choose the best operating point for the fan and the related nonlinear control such that the response to pressure variations is fast enough while the power consumption is low. In the analysis, we assume that the air flow is maintained in the laminar regime. In addition, we neglect the pneumatic capacitance effect of the enclosure, because we deal with small air quantities pumped for the pressure control. Large pressure drops are not considered here. The following notations are used throughout the paper. A basic pneumatic scheme is shown in Fig. 5, where symbols typically found in pneumatics were used. Figure 5 is explanatory for some of the symbols and equations described below. Here is an example of an inflatable enclosure supported by the control circuit:

$P_{a t m}$ atmospheric air pressure;

$P_{e}$ pressure inside the enclosure (absolute);

$P_{e m}$ minimal inside pressure required to maintain the structure walls; essentially $P_{e}-P_{a t} \geq m g / S_{u}$, where $m$ is the mass of the non-supported part of the enclosure (virtually, the entire enclosure), $g$ is the gravity constant, and $S_{u}$ is the surface of the (equivalent) upper wall of the enclosure, with the enclosure approximated with a prismatic box;

$P_{e M}$ maximal inside pressure allowed in the enclosure at normal (nominal) outside atmospheric pressure; depends on the material of the enclosure walls and the maximal forces exerted on the enclosure walls;

$q_{o} \quad$ optimal air flow $\mathrm{m}^{3} / \mathrm{min}$; equals optimal air flow from inside to outside the enclosure, at constant (static) pressure;

$P_{e o}$ optimal inside pressure in the enclosure, at nominal outside atmospheric pressure and at $q_{o}$;

$\Delta P_{e a}$ inside-outside (atmosphere) air pressure difference for the enclosure;

$\Delta P_{\text {eao }}$ optimal inside-outside air pressure difference for the enclosure;

$\eta \quad$ duty cycle of the PWM control;

$\Delta P_{a F}\left(q_{o}\right)$ pressure difference on the air filter at optimal air flow; typically, the pressure difference increases quadratically with $q$; precise curves are usually given by the filter manufacturers;

$\Delta P_{t}\left(q_{o}\right)$ pressure difference on the air tube (air path between filter and fan and between fan and enclosure) at optimal air flow;

$\Delta P_{f}(q)$ pressure difference on the fan; $\Delta P_{f}(q)=$ $\Delta P_{t}(q)+\Delta P_{a F}(q)+\Delta P(q)$

$\Delta P_{m f}\left(q_{o}\right)$ minimal pressure difference on the fan; this is the minimal static pressure required for the fan when $q_{o}=0$ (theoretical condition, does not apply to real use);
$\Delta\left(q_{o}\right)$ maximal pressure difference on the fan; this is the maximal static pressure allowed on the fan;

$\Delta P_{V}(q)$ pressure difference on an exhaust vane, at flow rate $P_{M f}$ through vane $q_{V}$

$P_{f}(U, \eta, q)$ is the static pressure produced by the fan when the supply voltage is $U$, the flow is $q$ and the duty factor is $\eta$.

The fan is a nonlinear device, when seen with the input at the motor voltage control and the output as pressure difference or flow. Also, the relationship between flow and pressure on the fan is nonlinear. Under nominal operation, it produces a static pressure, which is a function of the nominal voltage $U_{o}$ applied and the nominal flow $q_{0}$, thus implicitly depending on the atmospheric pressure $P_{a t m}$ for a specified $\Delta P_{o}$

$$
P_{f}\left(U_{o}, \eta, q_{o}\right)=P_{f}\left(\Delta P_{o}, P_{a t m}, q_{o}, U_{o}, \eta\right) .
$$

The above allows the derivation of the required value of the duty factor at nominal conditions, $\eta\left(\Delta P_{o}, P_{a t m}, q_{o}, U_{o}\right)$.

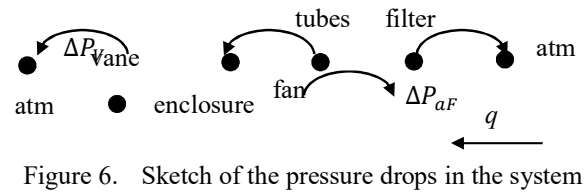

We will assume that there is no pressure drop inside the enclosure; this is a reasonable approximation for low flows, as the diameter of the enclosure (seen as a tube) is large. Therefore, $\Delta P_{e a}=\Delta P_{V}$. Along the air path, the following equation applies (see Fig. 6):

$$
P_{a t m}-\Delta P_{a F}-\Delta P_{t}-\Delta P_{e a}+\Delta P_{f}=P_{a t m},
$$

or, taking into account the dependence on the flow,

$$
\Delta P_{a F}(q)+\Delta P_{t}(q)+\Delta P_{e a}=\Delta P_{f}(q) .
$$

The values of $\Delta P_{a F}$ and $\Delta P_{t}$ depend on the filter and on the construction of the system; they can be assumed fixed, although $\Delta P_{a F}$ may increase over time due to the clogging of the filter over extended use.

The pressure difference $\Delta P_{V}(q)$ depends on the air flow $q$ when the flow is turbulent.

The maximal value allowed for $\Delta P_{V}(q)$ is $\Delta P_{V M}(q) \leq \Delta P_{e a}$, where the difference $\Delta P_{e a}-$ $\Delta P_{V M}(q)$ is larger than zero when there is a safety vane that let the air escape when internal pressure is high.

For equilibrium, the air flow produced by the fan should satisfy $q_{f}\left(P_{f}\right)=q_{V}$, assuming that the safety vane is closed and the flow through it, $q_{S V}$ is null. This condition, with the value $\Delta P_{f}=\Delta P_{a F}+\Delta P_{t}+$ $\Delta P_{e a}$ and $P_{a t m}$ determine the operation point of the fan. The fan control should satisfy the relationship

$$
\Delta P_{f}\left(U_{o}, \eta, q_{o}\right)=\Delta P_{f}\left(\Delta P_{o}, P_{a t m}, q_{o}, U_{o}, \eta\right)
$$


which, for a specified supply voltage $U_{o}$ determines $\eta$. As a matter of example, for an enclosure of mass of $1 \mathrm{~kg}$, with $g \approx 10 \mathrm{~m} / \mathrm{s}^{2}$ and the upper wall surface of $0.4 \mathrm{~m}^{2}, P_{e} \geq P_{a t m}+\frac{m g}{s_{u}}=P_{a t m}+2.5 \mathrm{~Pa}$. For a good deployment of the enclosure, a tenfold value (25 $\mathrm{Pa}$ in this example) is used as nominal pressure.

We now connect the previous discussion to the fan control. For a good control, we need a good model of the fan, expressed in terms of the pressure created as a function of air flow. As a working example, we assume a specific fan, SAN ACE 60, manufactured by Sanyo Denki. We also assume a power supply of 12 $\mathrm{V}$ and $100 \%$ duty cycle of the PWM control. With the graphical data from [7] approximated as in Table 1, for the fan type 9CR0612S001, at $12 \mathrm{~V}$, the second order polynomial approximation for the pressure $P$ as a function of air flow $q$ is reasonable and is

$$
P=-108.34 q^{2}+38.862 q+532.52
$$

$\left(\mathrm{R}^{2}=0.969\right)$, but the approximation is poor in the range of interest for the flow. For the same fan, the fifth order polynomial approximation is very good,

$$
\begin{gathered}
P=142.77 q^{5}-781.17 q^{4}+1388.4 q- \\
953.51 q^{2}+114.65 q+549.35
\end{gathered}
$$

with $\mathrm{R}^{2}=0.999$, see Fig. 7, while approximations of order 2, 3 and 4 are not acceptable for a fine control.

TABLE I. CHARACTERISTICS OF THE DISCUSSED FAN: FAN PRESSURE VS. FLOW

\begin{tabular}{|r|l|r|l|}
\hline $\begin{array}{l}\text { Air flow } \\
{\left[\mathrm{m}^{3} / \mathrm{min}\right]}\end{array}$ & $\begin{array}{l}\text { static pressure } \\
{[\mathrm{Pa}]}\end{array}$ & $\begin{array}{l}\text { Air flow } \\
{\left[\mathrm{m}^{3} / \mathrm{min}\right]}\end{array}$ & $\begin{array}{l}\text { static pressure } \\
{[\mathrm{Pa}]}\end{array}$ \\
\hline 0 & 550 & 1.5 & 380 \\
\hline 0.25 & 535 & 1.75 & 290 \\
\hline 0.5 & 500 & 2 & 150 \\
\hline 0.75 & 470 & 2.25 & 0 \\
\hline 1 & 460 & 2.5 & 0 \\
\hline 1.25 & 450 & & \\
\hline
\end{tabular}

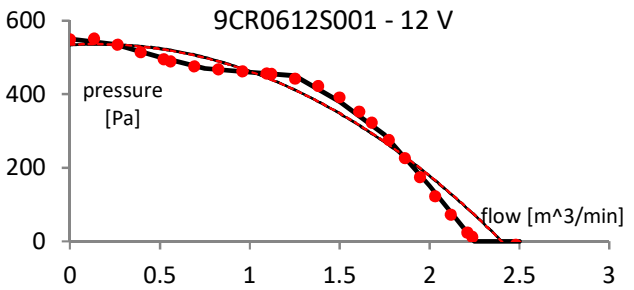

Figure 7. Approximation of the characteristic curve of one of the fans at $12 \mathrm{~V}$ operation (thick continuous line) by second (thin line) and fifth order (thick dotted line) polynomials, for $12 \mathrm{~V}$ power supply and $100 \%$ duty cycle of the PWM control.

Another model of fan, by the same manufacturer [7] has the characteristics in Fig. 8. For this type of fan, for $\eta=100 \%, 60 \%$ and $20 \%$, the approximations by quadratics are good. These approximations are: (i) For $=100 \%, \Delta P_{f}=$ $-316.48 q^{2}-345.56 q+1809.7,\left(\mathrm{R}^{2}=0.9987\right)$; (ii) For $\eta=60 \%, y=-209.52 x^{2}-228.57 x+$ $834.52,\left(\mathrm{R}^{2}=0.9962\right)$; (iii) For $\eta=20 \%, y=$ $-280 x^{2}-66 x+203.5\left(\mathrm{R}^{2}=0.9903\right)$, see Fig. 8 .

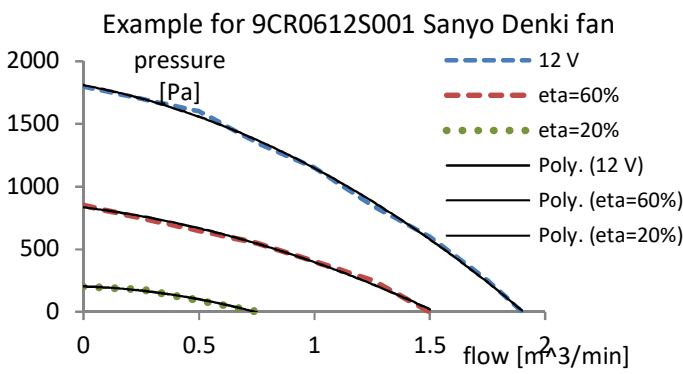

Figure 8. Characteristics of one of the fans, pressure vs. flow (thick lines), manually derived from the catalog, and the approximations with quadratics (thin lines almost superposed with the dotted lines representing the characteristic curves).

When the pressure in the enclosure drops from the nominal value, the pressure on the fan, $\Delta P_{e a}=P_{e}$ $P_{a t m}$ decreases, and as a consequence, the flow rate increases. Specifically, with $\Delta P_{f}(q)$ the pressure difference on the fan, $\Delta P_{f}(U, \eta, q)$, the static pressure produced by the fan when the supply voltage is $U$, the flow is $q$ and the duty factor is $\eta$,

$$
\Delta P_{a F}(q)+\Delta P_{t}(q)+\Delta P_{e a}=\Delta P_{f}(U, \eta, q),
$$

where, with $U$ and $\eta$ fixed, $q$ becomes a function of the other parameters. Assuming that $\Delta P_{a F}$ and $\Delta P_{t}$ do not change significantly with the air flow, $q=$ $q\left(\Delta P_{e a}\right)$. Relaxing the assumption of $q$ independence, to be more realistic, assume that $\Delta P_{a F}(q)=q \Delta P_{a F 0}$ and $\Delta P_{t}(q)=q \Delta P_{t 0} ;$ these are reasonable approximations. Then, the equation is

$$
q \Delta P_{a F 0}+q \Delta P_{t 0}+\Delta P_{e a}=\Delta P_{f}(U, \eta, q) .
$$

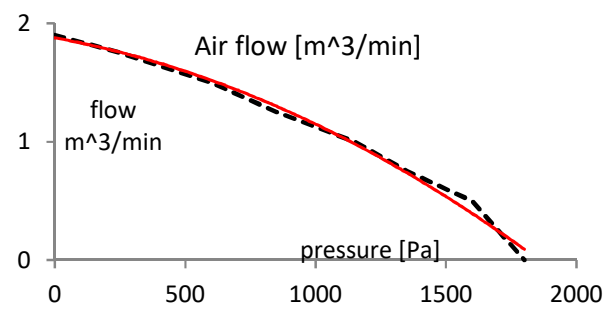

Figure 9. Characteristics of the fan in Fig 7, flow vs pressure (dotted line), and the approximations with quadratics.

The characteristic air flow vs. pressure on one of the fans is shown in Fig. 9. Notice that, when the pressure drops in the enclosure $\left(\Delta P_{e a} \downarrow\right), \Delta P_{f}$ at constant $U$ and $\eta$ also drops, with the effect of increasing $q$ until a new equilibrium is attained, according to the above equation. Therefore, the system has a natural tendency of stabilizing pressure in the enclosure, as a drop in enclosure pressure increases flow and replenish the enclosure, thus increasing pressure. The self-stabilization tendency is easily understood noticing that the derivative of the flow produced by the fan, $q_{f}\left(\Delta P_{f}\right)$ is negative, $\frac{\partial q_{f}}{\partial \Delta P_{f}}<0$. When this effect is not enough, for example because of a leak in the system, one may need to increase the value of $\eta$, as $\frac{\partial q_{f}}{\partial \eta}>0$. For the new model discussed, a 
good approximation of $q=q\left(\Delta P_{f}\right)$ is $q=-3$. $10^{-7}\left(\Delta P_{f}\right)^{2}-0.0004 \Delta P_{f}+1.877\left(\mathrm{R}^{2}=0.9935\right)$. When the variation with $q$ of the $\Delta P_{a F}$ and $\Delta P_{t}$ are introduced, one obtains:

$$
\begin{aligned}
& q \Delta P_{a F 0}+q \Delta P_{t 0}+\Delta P_{e a}=\Delta P_{f}=-316.48 q^{2}- \\
& 345.56 q+1809 .
\end{aligned}
$$

Equation (9) allows obtaining the flow for a specified set of tubes, and filter and for a given $\Delta P_{e a}$. This completes the set of design equations. Notice that any solution is allowed only when it satisfies the permitted range, for example the pressure inside the enclosure between $P_{e m}$ and $P_{e M}$. The above considerations show another role of the integrator used in the control loop: as long as the self-compensation is active and the decrease of the pressure in the enclosure is low, there is no need to increase the duty factor $\eta$. This behavior contributes to reducing the power of the system.

\section{DISCUSSION AND CONCLUSIONS}

The described system can be used for portable Continuous Positive Airway Pressure (CPAP) machines, BIPAPs (BI-level Positive Air Pressure), Powered Air-Purifying Respirators (PAPRs), ventilators and a set of other medical applications, including enclosures with airflow for patient isolation or surgery. In case of the current pandemic, there were many cases of asymptomatic subjects who spread the virus, which makes easy to modify and relatively inexpensive open design systems like the ones presented here of interest to those interested in increasing capacity of PAPRs for protection of medical providers.

As a limitation, we assumed throughout the analysis that the air flow is laminar, but this is not guaranteed at the higher values of the air flow, that is, for large values of the PWM duty cycle. All the analysis was done assuming that only small changes of pressure occur and that the changes are relatively slow, such that the pneumatic capacitances' effect of the enclosure and of the ducts are negligible. The case of fast and large swings in pressure in the enclosure requires modeling based on differential equations [8], [9], [10], [11] and is a topic for further research.

Because the suggested application devices often run on batteries, minimizing power losses in the circuit is important. Attention should also be paid to the use of fluid and dust resistive connectors (IP95 rated) and rock switches that allow the user to cut power from a battery or to cut power to the circuit of the fan at will. As higher amperage connectors have lower internal resistance, the voltage drop and the waste of power on such connectors is lower. For instance, it is preferable to have $10 \mathrm{~A}$ connectors; $5 \mathrm{~A}$ is minimal (but may cost less). All the circuits should withstand, under no operation, conditions up to $90{ }^{\circ} \mathrm{C}$, allowing disinfection with hot air at that temperature if needed.

Further work is needed for the optimization of the control and to combine pressure and flow control in a system aimed to reduce power consumption and to adapt the proposed circuit designs to the application.

\section{ACKNOWLEDGMENT}

This academic research has not been supported by any grant, company, or institution; the opinions regarding applicability are the authors' and not of their institutions. In accordance with norms of academic freedom, we provide these circuit designs as open designs, to allow for ease of further experimentation and improvements by the community.

DISCLAIMER: The systems and designs described here are for academic discussion and not intended to be used to treat any medical condition and, if the open designs presented here are adapted for any use, including medical device applications, they should be tested and certified in accordance with appropriate regulatory requirements in the country of use. The systems and designs in this paper are provided "as is", without warranty of any kind, express or implied, including but not limited to the warranties of suitability for a particular purpose, non-infringement, and merchantability. In no event shall the authors, their institutions, or copyright holders be liable for any claim, damages or other liability, whether in an action of contract, tort or otherwise, arising from, out of or in connection with the designs or the use or other dealings in the designs.

Authors' contribution: The authors contributed equally.

\section{REFERENCES}

[1] J.C. Taube, US5388575 Patent, Adaptive Controller for Automatic Ventilators, Feb. 14, 1995.

[2] C.B. Andreasen, US Patent 2,880,719, April 7, 1959, Lung ventilators and timing devices therefor.

[3] A. Battisti, D. Tassaux, J. Janssens, J.B. Michotte, S. Jaber, P. Jolliet, "Performance characteristics of 10 home mechanical ventilators in pressure-support mode." Chest, Vol. 127, no. 5, 2005, pp. 1784-92, https://doi.org/10.1378/chest.127.5.1784.

[4] D.L. Teodorescu et al., Patent application WO 2018/014003A1, Ultraportable System for Intraoperative Isolative and Regulation of Surgical Site Environments.

[5] A.M. Al Husseini et al., "Design and prototyping of a lowcost portable mechanical ventilator." Proc. 2010 Design of Medical Devices Conf. DMD2010, Apr 13-15, 2010, Minneapolis, MN, USA.

[6] C.R. MacIntyre, et al., "The first randomized, controlled clinical trial of mask use in households to prevent respiratory virus transmission." The Int. J. Infectious Diseases, Vol. 12, Supplement 1, e328, Dec. 01, 2008.

[7] Sanyo Denki Technical Report no. 44, Nov 2017. Available at https://www.sanyodenki.com/archive/document/corporatedata /technicalreport/2017/TR44_E.pdf.

[8] R.L. Chatburn, E. Mireles-Cabodevila.. "Chapter 3. Basic Principles of Ventilator Design." Principles and Practice of Mechanical Ventilation, 3e Ed. Martin J. Tobin. New York, NY: McGraw-Hill, 2013, http://accessmedicine.mhmedical.com/content.aspx?bookid $=5$ 20\&sectionid $=41692239$.

[9] N. Gehring, R. Kern, "Flatness-based tracking control for a pneumatic system with distributed parameters," IFACPapersOnLine, Vol. 51, no. 2, 2018, pp. 487-492, ISSN 24058963, https://doi.org/10.1016/j.ifacol.2018.03.082.

[10] E. Kondili, G. Prinianakis, D. Georgopoulos, "Patientventilator interaction." BJA: British J. Anaesthesia, Vol. 91, no. 1, 2003, pp. 106-119, https://doi.org/10.1093/bja/aeg129

[11] H. Güler, F. Ata, "Design and implementation of training mechanical ventilator set for clinicians and students." Procedia - Social and Behavioral Sciences 83 ( 2013 ) 493 496 1877-0428,2013 doi: 10.1016/j.sbspro.2013.06.095 